\title{
Variation de caractères agromorphologiques et du Brix d'une collection de sorghos à tige sucrée du Burkina Faso
}

\author{
Baloua NEBIE ${ }^{12 *}$, Romaric Kiswendsida NANEMA ${ }^{1}$, Pauline BATIONO/KANDO ${ }^{1}$, \\ Ernest Renan TRAORE ${ }^{1}$, Vanesse LABEYRIE ${ }^{3}$, Nerbéwendé SAWADOGO ${ }^{1}$, \\ Mahamadou SAWADOGO ${ }^{1}$ et Jean-Didier ZONGO ${ }^{1}$ \\ ${ }^{1}$ Laboratoire de Génétique et de Biotechnologie Végétales, Université de Ouagadougou 03 BP 7021 \\ Ouagadougou 03, Burkina Faso. \\ ${ }^{2}$ ICRISAT-Bamako, BP 320, Mali. \\ ${ }^{3}$ CIRAD, UMR AGAP, F-34398 Montpellier, France. \\ *Auteur correspondant, E-mail : nbaloua@yahoo.fr ; b.nebie@icrisatml.org ; \\ Tel : (+223)78132869/(+226)76006266
}

\section{RESUME}

Le sorgho à tige sucrée est de nos jours une plante d'intérêt utilisée pour la production de biocarburant. Au Burkina Faso, les ressources génétiques de ce sorgho ne sont pas connues, encore moins exploitées. Pour comprendre les relations entre la concentration en sucre des tiges et les caractères agromorphologiques de cette plante, 117 accessions ont été collectées dans quatre zones agro-climatiques du Burkina Faso. La caractérisation a été faite à partir de 15 variables quantitatives et de variables qualitatives qui discriminent pour la plupart les races de sorgho cultivé. Cette étude montre que les sorghos à tige sucrée du Burkina Faso présentent une diversité raciale plus importante en comparaison avec ceux d'autres pays comme le Mali et la Guinée. La majorité des accessions à nervure verte (77,21\% d'entre elles) ont des tiges juteuses jusqu'à la maturité physiologique des grains. Le Brix des tiges au stade grain dur varie entre 8,88 et $21,83 \%$. Il est négativement corrélé au rendement en grains par plante. Les accessions les plus sucrées sont celles à cycle long avec une importante biomasse. Les facteurs «zone climatique» et «race botanique» contribuent tous à expliquer la diversité observée avec cependant un effet plus important du facteur racial.

(c) 2013 International Formulae Group. All rights reserved.

Mots clés : accession, diversité agromorphologique, zone climatique, race.

\section{INTRODUCTION}

Le sorgho à tige sucrée appartient à l'espèce Sorghum bicolor (L.) Moench, du genre Sorghum et de la famille des Poaceae. Il regroupe différents écotypes dont la caractéristique commune est l'accumulation de quantités importantes d'hydrates de carbones dans leurs tiges juteuses (Ritter et al., 2008 ; Wang et al., 2013). Les principaux sucres contenus dans ce jus sont le saccharose, le glucose et le fructose, dans des proportions variables selon les variétés (Schaffer et Gourley, 1982; Murray et al., 2009). Selon Gutjahr (2012), les sorghos sucrés sont définis comme des sorghos de grande taille avec une importante biomasse. Ils sont peu productifs et ont des grains de mauvaise qualité. Ces sorghos sont cultivés dans plusieurs pays 
comme les Etats-Unis (Ali et al., 2007; Murray et al., 2009; Wang et al., 2009), le Niger (Deu et al., 2008), la Chine (Zhang et al., 2009 ; Pei et al., 2010), la Guinée et le Mali (Sagnard et al., 2011). Le sorgho à tige sucrée est traditionnellement cultivé en Afrique où leurs tiges sont consommées comme friandises et leurs grains sont utilisés dans l'alimentation humaine et animale. De nos jours, le sorgho à tige sucrée occupe une importance croissante dans l'industrie, car le jus sucré est utilisé pour produire du sirop et du bioéthanol. De ce fait, la caractérisation de la diversité agromorphologique et génétique du sorgho sucré représente un enjeu majeur pour l'amélioration variétale.

Longtemps classés dans le groupe des plantes mineures (Harlan et De Wet, 1972), les sorghos sucrés constituent une culture dont la diversité génétique reste peu connue. L'estimation de la concentration en sucre sur le terrain est faite à partir de la mesure du Brix qui est la fraction de matière sèche (principalement les sucres) dissoute dans le jus. La valeur du Brix dépend des variétés et est positivement corrélée à la hauteur de la plante (Murray et al., 2009; Zhang et al., 2009). Les variétés de sorgho à tige sucrée sont majoritairement de la race bicolor (Harlan et De Wet, 1972 ; Deu et al., 2008 ; Chantereau et al., 2010 ; Sagnard et al., 2011). Selon Pei et al. (2010), les sorghos à tige sucrée ont une diversité génétique modérée. Au Burkina Faso, les sorghos à tige sucrée ou «kankansiido» en langue mooré, n'ont pas encore été caractérisés, bien qu'ils y soient cultivés dans des conditions agro-écologiques variées. Malheureusement, comme la plupart des types marginaux, la culture des sorghos à tige sucrée est progressivement abandonnée. Leur préservation, valorisation et amélioration nécessitent une meilleure connaissance de leurs caractéristiques. Cette étude vise donc à caractériser la diversité agromorphologique des sorghos à tige sucrée du Burkina Faso, d'une part en identifiant les relations qui existent entre les caractères morphologiques et la concentration en sucre (Brix) des tiges, et d'autre part en décrivant la distribution de ces caractères en fonction des types raciaux et des zones agro-climatiques.

\section{MATERIEL ET METHODES}

\section{Site d'étude}

L'essai a été conduit en 2011 à Gampèla/ Burkina Fao, sur le site expérimental de l'Institut du Développement Rural (IDR) de l'Université Polytechnique de Bobo-Dioulasso $\left(1^{\circ} 21^{\prime}\right.$ de longitude Ouest et $12^{\circ} 24^{\prime}$ de latitude Nord). Cette station a bénéficié, du $1^{\text {er }}$ mai au 09 octobre 2011, d'une pluviométrie de $722,70 \mathrm{~mm}$.

\section{Matériel végétal}

Cent dix sept (117) accessions de sorgho à tige sucrée ont été collectées dans 65 villages, répartis dans les grandes zones agroclimatiques du Burkina Faso. Parmi ces accessions, 13 proviennent de la zone sahélienne stricte, 63 de la zone subsahélienne, 37 de la zone nord-soudanienne et 4 de la zone sud-soudanienne.

La collecte a été faite en collaboration avec les services techniques du Ministère de l'Agriculture et de la Sécurité Alimentaire du Burkina Faso. Les accessions ont été collectées auprès des paysans après renseignement d'une fiche qui mentionne les caractéristiques de chaque accession, son nom local ainsi que les pratiques culturales utilisées.

\section{Dispositif expérimental et conditions de culture}

Un dispositif en blocs de Fischer avec trois répétitions a été utilisé pour la caractérisation agromorphologique des accessions collectées. Il a été mis en place après un labour suivi d'un apport de fumure de fond (NPK 15-15-15) à la dose de 100 $\mathrm{Kg} / \mathrm{ha}$. Le semis a été effectué le 09 juillet 2011. Onze poquets ont été semés par entrée dans chaque répétition avec un écartement de $40 \mathrm{~cm}$ et un interligne de $80 \mathrm{~cm}$. Le semis a 
été suivi d'un démariage à un plant par poquet 14 jours après. Au stade gonflement de la panicule, une application de $50 \mathrm{~kg} /$ ha d'urée a été faite.

\section{Collecte et analyse des données}

Quinze (15) variables quantitatives ont été suivies dans chaque bloc. Le nombre de jours à la levée (NJL) et le nombre de jours à la floraison (NJF) ont été respectivement mesurés à $50 \%$ levée et à $50 \%$ floraison des pieds principaux de la ligne. Les autres variables quantitatives ont été mesurées à la maturité des grains sur 4 pieds aléatoirement choisis par ligne. Il s'agit du diamètre du 4 ième entre-nœud (DTI) à partir du sommet, de la longueur de l'entre-nœud (LOE) qui correspond à la demi-mesure de la distance entre le $3^{\text {ème }}$ et le $5^{\text {ème }}$ nœud, du nombre d'entre-nœuds (NEN), de la longueur du pédoncule (LPE), du poids frais de la tige (PTI), des longueurs de la panicule et de la $3^{\text {ième }}$ feuille sous le drapeau (LOP, LOF), de la largeur de la $3^{\text {ème }}$ feuille (LAF) ainsi que de la hauteur de la plante (HPL). Les caractères poids des panicules par poquet (PPO), rendement par plante (GPL), poids de cent grains (PCG) ont été également déterminés. La mesure du Brix a été réalisée à la maturité des grains, avec un réfractomètre numérique portable (ATAGO, PAL-alpha) de précision \pm $0,2 \%$. Une à deux gouttes (environ $0,3 \mathrm{ml}$ ) de jus de chacun des entre-nœuds $n^{\circ} 2,4$ et 6 ont été déposées dans la cellule de mesure de sorte à recouvrir le fond. En effet, Krishnaveni et al. (1984) et Bian et al. (2006) ont montré que le sucre est inégalement réparti au niveau des entre-nœuds d'une même tige, ce qui a guidé notre choix de trois différents entrenoeuds. La mesure du Brix de chaque individu correspond à la moyenne des valeurs obtenues pour chacun des trois entre-nœuds. En pressant la tige pour les mesures du Brix, nous avons également noté le caractère succulence de la tige pour préciser si la tige était juteuse ou sèche. Trois modalités (peu juteux, juteux et très juteux) ont été utilisées pour classer la succulence de la tige. La couleur de la nervure centrale a été également observée. La forme de la panicule et les caractéristiques de l'épillet (longueur des glumes, ouverture des glumes, forme et rotation du grain) ont été utilisées pour classer racialement les accessions étudiées selon la classification de Harlan et De Wet (1972).

Une analyse de variance (ANOVA) a été effectuée pour déterminer les caractères quantitatifs qui discriminent les accessions. Les relations entre ces caractères ont été étudiées grâce au test de corrélation de Pearson. Les caractères utilisés pour cette analyse sont le cycle, le rendement en grains par plante, le Brix et les caractères qui définissent la biomasse de la plante. Les effets principaux de la zone climatique et de la race botanique sur la variation des caractères quantitatifs ont été testés par une analyse de variance (ANOVA), suivi d'un test post-hoc de Tuckey pour tester la significativité des différences entre races et entre zones climatiques. Les données ont été analysées avec les logiciels XLSTAT-Pro 7.1 et R (RDCT, 2011).

\section{RESULTATS}

\section{Variation des caractères agromorphologiques et du Brix des sorghos à tige sucrée du Burkina Faso}

Le Tableau 1 présente les moyennes des caractères quantitatifs des sorghos à tige sucrée et certains paramètres de la dispersion des mesures. Le test d'ANOVA a révélé une différence significative entre les accessions au seuil de $1 \%$ pour tous les caractères étudiés, excepté pour le nombre de jours à la levée (NJL) et le diamètre de la tige (DTI).

Le cycle semis-floraison (NJF) varie de 57 à 98 jours avec une moyenne de 76 jours. Le nombre moyen d'entre-nœuds (NEN) est de 10,70 et leur longueur moyenne (LOE) de $19,54 \mathrm{~cm}$. Plus de la moitié des accessions $(55,55 \%)$ a une hauteur (HPL) supérieure à la hauteur moyenne de $233,71 \mathrm{~cm}$. Le poids frais de la tige principale (PTI) varie 
considérablement de 100 à 683,33 g selon les accessions, avec une moyenne de 357,72 g. Le rendement moyen en grains est de 34,20 $\mathrm{g} /$ plante et varie de 5,42 à 79,61 g. Quant à la mesure du Brix, elle varie de $8,88 \%$ à $21,83 \%$ avec une moyenne de $16,57 \%$. Soixante et onze accessions, soit un taux de $60,68 \%$ de la collection, ont un Brix supérieur à $16 \%$. Parmi ces accessions, 57 ont des tiges juteuses à très juteuses jusqu'au stade grain dur.

La plupart des caractères ont des coefficients de variation élevés. Les plus grands coefficients s'observent pour le rendement en grains par plante $(\mathrm{CV}=$ $36,81 \%)$ et le poids frais de la tige $(\mathrm{CV}=$ $33,81 \%)$.

Les accessions de la collection appartiennent à $56,41 \%$ à la race bicolor, $20,51 \%$ à la race caudatum, $17,10 \%$ à la race durra, et 5,98\% à la race bicolor-guinea. Elles ont pour la plupart des tiges juteuses à très juteuses au stade grain dur $(75,21 \%)$ et une nervure centrale verte $(82,91 \%)$. Les tiges de $77,21 \%$ des accessions à nervure verte sont juteuses à très juteuses et $35 \%$ des accessions à nervure blanche sont peu juteuses.

Relations entre les caractères quantitatifs des sorghos à tige sucrée du Burkina Faso

Les corrélations entre les caractères quantitatifs sont pour la plupart significatives au seuil de 5\% (Tableau 2). La corrélation qui existe par exemple entre la hauteur de la plante (HPL) et la mesure du Brix est significative et positive avec un coefficient de corrélation $r=0,345$. La hauteur de la plante est également positivement corrélée au poids frais de la tige $(r=0,353)$, et au nombre d'entre-nœuds ( $r=0.193$ ). Le cycle semisfloraison (NJF) est aussi significativement et positivement corrélé au poids frais de la tige (PTI) ( $\mathrm{r}=0,361)$, au nombre d'entre-nœuds $(r=0,744)$ et à la mesure du Brix $(r=0,235)$. Il en est de même pour le Brix et le nombre d'entre-nœuds (NEN) $(r=0,309)$. En revanche, le Brix, le cycle semis-floraison et la hauteur des plantes sont chacun significativement et négativement corrélé au rendement en grains par plante (GPL) avec respectivement des coefficients de corrélation de $-0,339 ;-0,399$ et $-0,250$.

Effet des facteurs «zone climatique » et « race botanique» sur la variation des caractères des sorghos à tige sucrée du Burkina Faso

Les $\mathrm{F}$ values du Tableau 3 montrent que la zone climatique a un effet significatif sur l'expression de 11 des 15 caractères quantitatifs étudiés qui sont entre autres le cycle semis-floraison (NJF), le Brix, la hauteur de la plante (HPL), la longueur des entre-nœuds (LOE), le rendement en grains par plante (GPL), le poids de cent grains (PCG). La race, quant à elle, a un effet hautement significatif ( $\mathrm{F}$ value $<1 \%$ ) sur l'expression de tous les caractères qui discriminent les accessions.

Les coefficients de détermination montrent que le facteur « race botanique» explique par exemple $69,17 \%$ de la variance de la longueur des panicules, 49,00\% de la largeur des feuilles, 46,98\% de la longueur du pédoncule, $36,09 \%$ de la hauteur des plantes, $27,64 \%$ du rendement en grains par plante et $22,96 \%$ de la longueur des feuilles.

Les valeurs $\mathrm{du}$ coefficient de détermination $\mathrm{du}$ facteur «zone climatique » sont par contre faibles dans l'ensemble $(<20 \%)$ excepté pour le poids de cent grains $\left(\mathrm{R}^{2}=34,97 \%\right)$. Le facteur zone climatique explique mieux la variance du poids de cent grains, du cycle semis-floraison $\left(\mathrm{R}^{2}=14,60 \%\right)$ et du poids frais de la tige $\left(\mathrm{R}^{2}=14,08 \%\right)$ que le facteur race $(5,60 \%$, $11,56 \%$ et $12,10 \%)$. Les facteurs «zone climatique » et « race botanique » expliquent tous deux la variance du Brix avec cependant de faibles coefficients de détermination. Cette variance semble être mieux expliquée par le facteur race botanique $(11,39 \%$ contre $7,94 \%)$. 
Tableau 1: Valeurs des 15 caractères quantitatifs et description de la diversité des sorghos à tige sucrée.

\begin{tabular}{lccccc}
\hline Variables & Mini. & Max. & Moy. & C V (\%) & F \\
\hline NJL $(\mathrm{j})$ & 3,00 & 4,50 & 3,22 & 10,32 & 1,153 \\
NJF $(\mathrm{j})$ & 57,33 & 98,00 & 76,45 & 7,36 & $4,210^{* *}$ \\
LOF $(\mathrm{cm})$ & 46,31 & 85,63 & 70,84 & 10,22 & $6,811^{* *}$ \\
LAF $(\mathrm{cm})$ & 5,30 & 11,00 & 7,82 & 19,49 & $7,838^{* *}$ \\
NEN & 6,33 & 15,67 & 10,70 & 15,92 & $9,366^{* *}$ \\
LOE $(\mathrm{cm})$ & 8,71 & 29,25 & 19,54 & 24,86 & $11,456^{* *}$ \\
HPL $(\mathrm{cm})$ & 126,25 & 344,38 & 233,71 & 18,94 & $11,452^{* *}$ \\
DTI $(\mathrm{cm})$ & 1,24 & 2,23 & 1,59 & 12,23 & 0,887 \\
LOP $(\mathrm{cm})$ & 10,83 & 42,42 & 26,24 & 27,68 & $12,194 * *$ \\
LPE $(\mathrm{cm})$ & 20,22 & 78,00 & 42,58 & 27,67 & $12,587 * *$ \\
PTI $(\mathrm{cm})$ & 100,00 & 683,33 & 357,72 & 33,81 & $2,318^{* *}$ \\
PPO $(\mathrm{g})$ & 15,79 & 104,13 & 58,35 & 28,80 & $3,396^{* *}$ \\
GPL $(\mathrm{g})$ & 5,42 & 79,61 & 34,20 & 36,81 & $3,619^{* *}$ \\
PCG $(\mathrm{g})$ & 1,24 & 3,25 & 2,24 & 17,56 & $5,174 * *$ \\
Brix $(\%)$ & 8,88 & 21,83 & 16,57 & 16,39 & $3,819^{* *}$ \\
\hline
\end{tabular}

NJL : nombre de jours à la levée, NJF : nombre de jours à la floraison, LOF : longueur de la $3^{\text {ieme }}$ feuille sous paniculaire, LAF : largeur de la $3^{\text {ième }}$ feuille sous paniculaire, NEN : nombre d'entre-nœuds, LOE : longueur de l'entre-nœud, HPL: hauteur de la plante, DTI : diamètre de la tige principale, LOP : longueur de la panicule, LPE : longueur du pédoncule, PTI : poids frais de la tige, PPO : poids des panicules par poquet, GPL : rendement en grains par plante, PCG : poids de cent grains, Brix : concentration de solutés dans le jus, Min. : minimum, Max. : maximum, Moy. : moyenne, CV : coefficient de variation, $\mathrm{F}$ : coefficient de Fischer, ** : différence significative à $1 \%$.

Tableau 2 : Corrélations entre huit caractères quantitatifs des sorghos à tige sucrée du Burkina Faso.

\begin{tabular}{lccccccc}
\hline & NJF & LOF & LAF & NEN & HPL & PTI & GPL \\
\hline LOF & $-0,13$ & 1 & & & & & \\
LAF & 0,102 & $0,398^{*}$ & 1 & & & & \\
NEN & $0,744^{*}$ & $-0,462^{*}$ & 0,043 & 1 & & & \\
HPL & $-0,01$ & $-0,285^{*}$ & $-0,531^{*}$ & $0,193^{*}$ & 1 & & \\
PTI & $0,361^{*}$ & $-0,377^{*}$ & $-0,022$ & $0,556^{*}$ & $0,353^{*}$ & 1 & \\
GPL & $-0,399^{*}$ & $0,463^{*}$ & $0,499^{*}$ & $-0,320^{*}$ & $-0,250^{*}$ & $-0,174$ & 1 \\
Brix & $0,235^{*}$ & $-0,04$ & $-0,178$ & $0,309^{*}$ & $0,345^{*}$ & 0,128 & $-0,339^{*}$
\end{tabular}

* : corrélation significative au seuil de $5 \%, \mathrm{NJF}$ : nombre de jours à la floraison, LOF : longueur de la $3^{\text {ieme }}$ feuille sous paniculaire, LAF : largeur de la $3^{\text {ieme }}$ feuille sous paniculaire, NEN : nombre d'entre-nœuds, HPL: hauteur de la plante, PTI : poids frais de la tige, GPL : rendement grain par plante, Brix : concentration de solutés dans le jus. 
Tableau 3: Comparaison des effets de la zone climatique et de la race sur l'expression des caractères des sorghos à tige sucrée du Burkina Faso.

\begin{tabular}{lcccc}
\hline Caractères & F value (z. cli) & F value (race) & $\mathbf{R}^{2}$ z. cli $(\mathbf{e n} \%)$ & $\mathbf{R}^{2}$ race (en \%) \\
\hline NJL $(\mathrm{j})$ & 0,30765671 & 0,8211489 & 3,18 & 0,80 \\
NJF $(\mathrm{j})$ & $0,000175278^{* *}$ & $0,001099356^{* *}$ & 14,60 & 11,56 \\
LOF $(\mathrm{cm})$ & $0,039202323^{*}$ & $9,36 \mathrm{E}-07^{* *}$ & 5,61 & 22,96 \\
LAF $(\mathrm{cm})$ & 0,566378096 & $2,99 \mathrm{E}-16^{* *}$ & 0,93 & 49,00 \\
NEN & 0,117452265 & $3,21 \mathrm{E}-06^{* *}$ & 4,06 & 21,65 \\
LOE $(\mathrm{cm})$ & $0,000903126^{* *}$ & $1,07 \mathrm{E}-12^{* *}$ & 8,63 & 37,70 \\
HPL $(\mathrm{cm})$ & $2,41 \mathrm{E}-06^{* *}$ & $4,06 \mathrm{E}-13^{* *}$ & 14,69 & 36,09 \\
DTI $(\mathrm{cm})$ & 0,945872593 & $4,73 \mathrm{E}-12^{* *}$ & 0,20 & 39,55 \\
LOP $(\mathrm{cm})$ & $0,004626001^{* *}$ & $5,90 \mathrm{E}-30^{* *}$ & 3,42 & 69,17 \\
LPE $(\mathrm{cm})$ & $4,46 \mathrm{E}-08^{* *}$ & $3,52 \mathrm{E}-19^{* *}$ & 15,11 & 46,98 \\
PTI $(\mathrm{g})$ & $0,000238083^{* *}$ & $0,000784988^{* *}$ & 14,08 & 12,10 \\
PPO $(\mathrm{g})$ & $0,000280295^{* *}$ & $0,000112088^{* *}$ & 13,41 & 14,90 \\
GPL $(\mathrm{g})$ & $0,005893579^{* *}$ & $1,47 \mathrm{E}-08^{* *}$ & 7,73 & 27,64 \\
PCG $(\mathrm{g})$ & $4,61 \mathrm{E}-11^{* *}$ & $0,018976269^{* *}$ & 34,97 & 5,60 \\
Brix $(\%)$ & $0,015612981^{*}$ & $0,002199566^{* *}$ & 7,94 & 11,39 \\
\hline
\end{tabular}

NJL : nombre de jours à la levée, NJF : nombre de jours à la floraison, LOF : longueur de la $3^{\text {ième }}$ feuille sous paniculaire , LAF : largeur de la $3^{\text {ième }}$ feuille sous paniculaire, NEN : nombre d'entre-nœuds, LOE : longueur de l'entre-nœud, HPL: hauteur de la plante, DTI : diamètre de la tige principale, LOP : longueur de la panicule, LPE : longueur du pédoncule, PTI : poids frais de la tige, PPO : poids des panicules par poquet, GPL : rendement en grains par plante, PCG : poids de cent grains, Brix : concentration de solutés dans le jus, Z. cli : zone climatique, $*$ : différence significative à $5 \%$, ** : différence significative à $1 \%, \mathrm{R}^{2}$ : coefficient de détermination.

\section{DISCUSSION}

Variation des caractères des sorghos à tige sucrée du Burkina Faso

L'analyse de variance a mis en évidence une importante diversité phénotypique des sorghos à tige sucrée, surtout pour le poids frais de la tige, la longueur de la panicule et les paramètres du rendement (poids des panicules par poquet et rendement en grains par plante) qui ont montré des coefficients de variation élevés. Djè et al. (2006) ont également obtenu de forts coefficients de variation pour le poids et la longueur des panicules de variétés traditionnelles de sorgho au Maroc.

La variation des mesures du Brix $(8,88$ à $21,83 \%$ ) est similaire aux résultats de Ali et al. (2007), Makanda et al. (2009), Zhang et al. (2009), Gutjahr (2012) qui sont respectivement 7,13 à $19,26 \% ; 6,48$ à $20,68 \% ; 8$ à $22 \%$ et 6,1 à $21,6 \%$. La collection étudiée a par contre des mesures de Brix plus variées que celles caracérisées par
Channappagoudar et al. (2007) (11 à 16,3\%), Ritter et al. (2008) (7,42 à 14,4\%), Hunsigi et al. (2010) (11,4 à 17\%), Kim et Day (2010) (11 à 13\%). La grande variation d'accumulation de sucre de nos accessions témoigne de l'hétérogénéité de la collection étudiée. Cela pourrait être lié à la forte diversité raciale des sorghos à tige sucrée du Burkina Faso. En plus des différentes races (excepté kaffir) identifiées par Murray et al. (2009) aux Etats-unis, il existe au Burkina Faso des sorghos à tige sucrée de la race intermédiare bicolor-guinea. En effet, Murray et al. (2009) ont identifié trois groupes de sorgho sucré qui sont les "variétés sucre et énergie » (race caudatum) caractérisées par une forte teneur en sucre, les «variétés sirop » (races kaffir/bicolor) avec des tiges de grande taille, juteuses mais moins sucrées que les premières et les «variétés amber» (races durra et bicolor) dont la teneur en sucre varie selon les milieux. La différence entre nos résultats et ceux de certains auteurs 
mentionnés ci-dessus pour le Brix peut s'expliquer par le type de matériel génétique étudié ou peut être due à la taille des échantillons. Ritter et al. (2008) ont par exemple étudié une population de 184 lignées mais à base génétique restreinte. Cette population est issue de croisement en $\mathrm{F}_{6}$ d'un sorgho sucré et d'un sorgho non sucré. Gutchjar et al. (2012) par contre ont analysé 14 génotypes de sorgho sucré avec cependant une riche diversité raciale. Le stade de récolte des tiges pour les mesures du Brix pourrait également expliquer les différences de valeurs obtenues. En effet, le Brix augmente avec les stades de développement du sorgho. Il est plus élevé à la maturité physiologique des grains (Krishnaveni et al., 1984 ; Muminov, 1997 ; Channappagoudar et al., 2007 ; Chavan et al., 2009).

Les accessions de grande taille, avec de nombreux entre-nœuds et un cycle long ont en général un Brix élevé. Elles ont par contre un faible rendement en grains. Ces résultats peuvent s'expliquer par la physiologie de la plante. Les accessions qui ont un long cycle de développement édifient de grands organes végétatifs capables de stocker des quantités considérables de matière sèche. Elles parviennent donc à accumuler plus de sucre dans les tiges lorsque les conditions pluviométriques sont favorables et ce, parfois au détriment des grains. L'accumulation des sucres semble liée à la capacité de la plante à développer une importante biomasse. Ritter et al. (2008), Murray et al. (2009) et Gutjahr (2012) ont également obtenu des corrélations significatives et positives entre le Brix, la hauteur de la plante, le poids sec de la tige et le cycle. La corrélation entre le Brix et le rendement en grains était par contre négative (Ritter et al., 2008). Selon ces mêmes auteurs, au moins deux QTLs situés sur le chromosome 6 interviennent dans la régulation du cycle, de la hauteur de la plante et du Brix. Si ces QTLs ont un effet additif pour chacun des caractères, cela permettrait de comprendre les corrélations que nous avons obtenues entre ces différents caractères. Ils n'ont par contre trouvé aucun QTL commun au rendement en grains et à chacun des caractères mentionnés ci-dessus. Cependant, la relation entre la concentration en sucre et le rendement en grains reste controversée. Elle dépend également des variétés. Certains génotypes de la collection ont un Brix supérieur à $16 \%$ avec cependant un bon rendement en grains. Gutjahr (2012) n'a trouvé aucun lien direct entre le Brix et le rendement en grains. Il a cependant obtenu une corrélation négative entre le Brix et l'indice de récolte. Le Brix à la maturité du grain était fortement influencé par l'interaction date de semis-environnement. Selon Makanda et al. (2009), le Brix à 50\% floraison est significativement et positivement corrélé au rendement en grains, tandis que la corrélation entre le Brix à maturité physiologique du grain et la hauteur de la plante est négative.

La majorité des accessions de la collection qui ont un Brix élevé ont également des tiges juteuses et des feuilles à nervure principale verte. Certaines accessions associent à ces caractères un rendement en grains moyen. Ces accessions rassemblent bien les critères de sélection recherchés chez les sorghos sucrés. Dans un pays comme le Burkina Faso, où le sorgho est cultivé pour ses grains, les variétés de sorgho à tige sucrée capables de produire des rendements acceptables en jus et en grains avec cependant une bonne teneur en sucre seront les plus appréciés des producteurs. Quelques accessions à nervure blanche ont aussi un Brix élevé mais sont peu juteux. La couleur de la nervure informe sur la succulence de la tige mais la différence entre la couleur verte et blanche n'est pas toujours évidente.

Selon Nebié (2009), le sorgho à tige sucrée est produit au Burkina Faso dans les champs de case et en association avec d'autres cultures (maïs, sorgho non sucré, mil). Ces pratiques culturales qui favorisent le brassage génétique entre le sorgho sucré et le sorgho non sucré contribueraient à expliquer la riche diversité raciale des sorghos à tige sucrée du Burkina Faso. Parmi les races identifiées, la race bicolor est la plus dominante. Les races 
caudatum, durra et bicolor-guinea y ont été également identifiées. Cette diversité raciale est similaire à celle observée au sein des sorghos à tige sucrée du Niger (Deu et al., 2008). Elle est même plus importante que la diversité observée au Mali. Selon Sagard et al. (2011) la race bicolor est la seule race principale des sorghos à tiges sucrée du Mali. D'autres races y sont représentées mais à travers des hybrides.

La diversité inter-raciale au sein des sorghos à tige sucrée du Burkina Faso est particulièrement importante. Toutes les races, excepté la race bicolor-guinea (7 accessions) ont un effectif supérieur ou égal à 20 accessions. Dans ce matériel, il existe des accessions à forte concentration en sucre avec un rendement en grains moyen. Les graines de certaines de ces accessions sont de bonne qualité (au moins la moitié de l'albumen est vitreuse). Cette diversité des sorghos à tige sucrée du Burkina Faso pourrait être exploitée par les sélectionneurs dans les programmes de sélection pour créer des variétés à multiples usages.

Influence des facteurs «zone climatique» et « race botanique» sur la variation des caractères des sorghos à tige sucrée au Burkina Faso

La zone climatique influence l'expression des variables quantitatives des sorghos à tige sucrée mais cette influence est moins importante que celle du facteur « race botanique ». Selon Haussmann et al. (2011), grâce à la plasticité phénotypique, les génotypes cultivés dans des conditions pluviométriques diversifiées s'adaptent aux contraintes du milieu par la réduction ou l'augmentation de certains caractères comme le cycle, la biomasse, etc. Ce qui explique l'influence de la zone climatique constatée sur la durée du cycle, le poids frais de la tige et le poids de cent grains. Les races quant à elles constituent des types botaniques définis sur la base de certains caractères morphologiques (Harlan et De Wet, 1972). Il n'est donc pas étonnant que celles-ci expliquent mieux la diversité observée. Les accessions de la race caudatum sont généralement de petite taille par rapport aux bicolor et bicolor-guinea. L'accumulation du sucre étant liée à la biomasse de la plante (Gutjahr, 2012), cela explique l'effet hautement significatif du facteur race botanique sur les mesures du Brix. Cependant la faible influence de la zone climatique sur la variation de la concentration en sucre (Brix) est surprenante. En effet, la zone climatique est l'un des principaux facteurs qui influencent le cycle de la plante qui à son tour favorise l'édification des organes végétatifs. On s'attendrait à ce que les accessions collectées dans les différentes zones climatiques (pluviométrie $<500 \mathrm{~mm}$ à $1100 \mathrm{~mm}$ ) aient des Brix très différents, ce qui se traduirait par un effet hautement significatif $\mathrm{du}$ facteur zone climatique sur le Brix. L'influence de la zone climatique sur la variation de la durée du cycle et du poids de cent grains est hautement significative mais seulement significative sur les mesures de Brix. Zongo (1991) a également obtenu avec les sorghos traditionnels du Burkina Faso, composés essentiellement de bicolor-guinea, une influence plus importante de la zone climatique sur la variabilité du cycle par rapport à celle de la race botanique mais la variance du poids de cent grains était surtout expliquée par la race. Selon Barro/Kondombo (2010), la zone climatique explique la variance du cycle avec un coefficient de détermination de $27,8 \%$ mais l'effet du facteur «variété » était plus important $(84,8 \%)$. L'effet de ces différents facteurs sur la variation du Brix et des caractères agromorphologiques est complexe et cela pourrait être mieux élucidé à l'aide d'essais multilocaux.

\section{Conclusion}

Notre étude a permis d'identifier un important nombre de caractères agronomiques et morphologiques pour la caractérisation des sorghos à tige sucrée. Ce groupe de sorgho a une riche diversité raciale et une large variabilité d'accumulation des sucres dans les tiges. Le Brix est négativement corrélée au rendement en grains mais positivement 
corrélée au cycle, à la hauteur de la plante et au nombre d'entre-nœuds. Certaines accessions associent cependant Brix élevé, tige juteuse et rendement en grains moyen. L'importante diversité mise en évidence au niveau agromorphologique offre un large potentiel pour l'amélioration variétale du sorgho. Cette diversité pourrait être valorisée pour l'alimentation, le bioéthanol, le fourrage ainsi que pour le développement de variétés multiples usages. Une bonne adéquation entre la classification raciale et la structure génétique est observée. Il a également été mis en évidence une influence de la diversité agromorphologique par le facteur zone climatique. L'action du climat, de la race et d'autres facteurs importants serait mieux établie à partir d'effectifs plus équilibrés des différentes zones agro-climatiques et d'essais multi-locaux. L'étude de la diversité génétique des sorghos à tige sucrée du Burkina Faso, à travers les marqueurs moléculaires, permettrait d'approfondir notre connaissance de ce pool génétique qui n'avait jusqu'à présent pas été caractérisé. Les liens entre groupes ethniques et diversité génétique pourraient également être étudiés pour une meilleure conservation et valorisation des sorghos à tige sucrée du Burkina Faso.

\section{RÉFÉRENCES}

Ali ML, Rajewski JF, Baenziger PS, Gill KS, Eskridge KM, Dweikat I. 2007. Assessment of genetic diversity and relationship among a collection of US sweet sorghum germplasm by SSR markers. Molecular Breeding, 21: 497509.

Barro/Kondombo C. 2010. Diversité agromorphologique et génétique de variétés locales de sorgho (Sorghum bicolor (L.) Moench) au Burkina Faso. Eléments pour la valorisation des ressources génétiques locales. Thèse de doctorat, Univ. de Ouagadougou, p. 112.

Bian Y, Yazaki S, Inoue M, Cai H. 2006. QTLs for sugar content of stalk in sweet sorghum (Sorghum bicolor L. Moench).
Agricultural Sciences in China, 5(10): 736-744.

Chantereau J, Deu M, Pham JL, Kapran I, Vigouroux Y Bezançon G. 2010. Evolution des diversités phénotypique et génétique des sorghos et mils cultivés au Niger de 1976 à 2003. Le Sélectionneur Français, 61: 33-45.

Channappagoudar BB, Biradar NR, Patil JB, Hiremath SM. 2007. Assessment of sweet sorghum genotypes for cane yield, juice characters and sugar levels. Karnataka J. Agric. Sci., 20(2): 294-296.

Chavan UD, Patil JV, Shinde MS. 2009. An assessment of sweet sorghum cultivars for ethanol production. Sugar Tech., 11(4): 319-323.

Deu M, Sagnard F, Chantereau J, Calatayud C, Hérault D, Mariac C, Pham JL, Vigouroux Y, Kapran I, Traoré PS, Mamadou A, Gérard B, Ndjeunga J, Bezançon G. 2008. Niger-wide assessment of in situ sorghum genetic diversity with microsatellite markers. Theor. Appl. Genet., 116: 903-916.

Djè Y, Heuertz M, Ater M, Lefebvre C, Vekemans X. 2006. Évaluation de la diversité morphologique des variétés traditionnelles de sorgho du Nord-ouest du Maroc. Biotechnol. Agron. Soc. Environ., 11(1): 39-46.

Gutjahr S. 2012. Analyse des caractères d'intérêt morphogénétiques et biochimiques pour le développement de sorghos sucrés à double usage grainbioalcool . Thèse de doctorat, Université Montpellier 2, France, p. 112.

Harlan JR, De Wet JMJ. 1972. Une classification simplifiée des sorghos cultivés. Crop Science, 9(2): 172-176.

Haussmann BIG, Rattunde HF, WeltzienRattunde E, Traoré PSC, vom Brocke K, Parzies HK. 2012. Breeding Strategies for Adaptation of Pearl Millet and Sorghum to Climate Variability and Change in West Africa. Journal of Agronomy and Crop Science, 198: 327-339.

Hunsigi G, Yekkeli NR, Kongawad BY. 2010. Sweet stalk sorghum: an alternative sugar 
crop for ethanol production. Sugar. Tech., 12(1): 79-80.

Kim M, Day DF. 2010. Composition of sugar cane, energy cane, and sweet sorghum suitable for ethanol production at Louisiana sugar mills. J. Ind. Microbiol. Biotechnol., 38(7): 803-807.

Krishnaveni S, Balasubramanian T, Sadasivam S. 1984. Sugar Distribution in Sweet Stalk Sorghum. Food Chemistry, 15: 229-232.

Makanda I, Tongoona P, Derera J. 2009. Quantification of genotypic variability for stem sugar accumulation and associated traits in new sweet sorghum varieties. African Crop Science Conference Proceedings, 9: 391-398.

Muminov NS. 1997. Dynamic of the accumulation of sugars in sweet sorghum. Chemistry of Natural Compounds, 33(3): 353-354.

Murray SC, Rooney WL, Hamblin MT, Mitchell SE, Kresovich S. 2009. Sweet sorghum genetic diversity and association mapping for brix and height. The Plant Genome, 2(1): 15.

Nebié B. 2009. Etude de la variabilité agromorphologique de quelques écotypes de sorghos sucres [Sorghum bicolor (L.) Moench] du Burkina Faso. Mémoire de DEA, Université de Ouagadougou, p. 62.

Pei Z, Gao J, Chen Q, Wei J, Li Z, Luo F, Shi L, Ding B, Sun S. 2010. Genetic diversity of elite sweet sorghum genotypes assessed by SSR markers. Biologia Plantarum, 54(4): 653-658.

RDCT (R Development Core Team). 2011. $R$ : A Language and Environment for Statistical Computing. R Foundation for Statistical Computing: Vienna, Austria:

Ritter KB, Jordan DR, Chapman SC, Godwin ID, Mace ES, Mcintyre CL. 2008.
Identification of QTL for sugar-related traits in a sweet $\mathrm{x}$ grain sorghum (Sorghum bicolor L. Moench) recombinant inbred population. Mol. Breeding, 22: 367-384.

Sagnard F, Deu M, Dembélé D, Leblois R, Touré L, Diakité M, Calatayud C, Vaksmann M Bouchet S, Mallé Y, Togola S, Traoré PCS. 2011. Genetic diversity, structure, gene flow and evolutionary relationships within the Sorghum bicolor wild-weedy-crop complex in a western African region. Theor. Appl. Genet., 123(7): 1231-1246.

Schaffer RE, Gourley LM. 1982. Sorghum as an energy source. In Sorghum in The Eighties, House LR, Mughogho LK, Peack JM (eds). ICRISAT: Patancheru, Inde; 477-783.

Wang ML, Zhu C, Barkley NA, Chen Z, Erpelding JE, Murray SC, Tuinstra MR, Tesso T, Pederson GA, Yu J. 2009. Genetic diversity and population structure analysis of accessions in the US historic sweet sorghum collection. Theor. Appl. Genet., 120: 13-23.

Wang L, Jiao S, Jiang Y, Yan H, Su D, Sun G, Yan X, Sun L. 2013. Genetic diversity in parent lines of sweet sorghum based on agronomical traits and SSR markers. Field Crops Research., 149: 11-19.

Zhang C, Xie G, Li S, Ge L, He T. 2009. The productive potentials of sweet sorghum ethanol in China. Applied Energy, 87: 2360-2368.

Zongo JD. 1991. Ressources génétiques des sorghos (Sorghum bicolor L. Moench) du Burkina Faso: Evaluation agromorphologique et génétique. Thèse de Doctorat d'état, Université d'Abidjan, Côte d'Ivoire, p. 175. 